\title{
Zur Genese der Hönninger Sande (unterer Mittelrhein)
}

\author{
Wolfgang Boenigk \& Christian Hoselmann *)
}

\author{
Pleistocene, stratigraphy, terraces, sand, heavy minerals, \\ reworked sands, moment measures, standard deviation, normal distribution \\ Lower Middle Rhine Valley, Rhenish Massif \\ TK 25 Nr.: 5408, 5409, 5410
}

\begin{abstract}
Kurzfassung: Im Gebiet des unteren Mittelrheins wurden mehrere Sandvorkommen im Bereich der älteren Hauptterrasse untersucht. Diese Sandfazies, die eine Mächtigkeit von bis zu $30 \mathrm{~m}$ erreichen kann, wird insgesamt als Hönninger Sand bezeichnet. Die Hönninger Sande werden mit den Mosbacher Sanden und zwar mit dem mittleren und oberen Teil des „Mittleren” bzw. „Grauen” Mosbachs bei Wiesbaden korreliert. Damit erfolgt eine Einstufung der älteren Hauptterrassen am unteren Mittelrhein in den CromerKomplex i.w.S.
\end{abstract}

In dieser Arbeit wird eine differenziertere Untergliederung der Sandvorkommen vorgeschlagen. Nur der jeweils untere Teil der Profile ist als fluviatile Hönninger Sande i.e.S. zu verstehen. Die sehr mächtigen Schichtenfolgen entstehen durch Umlagerung älterer Sedimente u. a. der Hönninger Sande. Diese werden als Linzer Sande bezeichnet. Die Umlagerung erfolgte durch Solifluktion sowie fluviatilen und äolischen Transport. Eine wichtige Konsequenz ist, daß die meisten Sandvorkommen in der Höhenlage der älteren Hauptterrasse keinen stratigraphischen Leitwert besitzen.

Die statistische Auswertung erfolgte durch die Berechnung der Momente. Hier sind vor allem die Standardabweichung und das arithmetische Mittel eine geeignete Methode um ein Sediment zu charakterisieren. Die Korngrößenanalysen zeigten, daß diese gut bis sehr gut sortierten Sande einen Siebabstand von 0,125 Zeta erfordern, um die Gauß'schen Regeln zur Darstellung von Normalverteilungen im Wahrscheinlichkeistnetz zu erfüllen. Für den Sandbereich erhält man so 12 Siebklassen.

\section{[The Genesis of the Hönninger Sands (Lower Middle Rhine Valley)]}

Abstract: In the area of the Lower Middle Rhine Valley serveral sand deposits within the older main terrace have

*) Anschriften der Verfasser: Prof. Dr. W. BoENIGK, Dipl.-Geol. CH. HoselmanN, Abt. Quartärgeologie, Geologisches Institut der Universität zu Köln, Zülpicher Str. 49, D - 5000 Köln 1. been investigated. This sand facies which can reach a thickness up to $30 \mathrm{~m}$ is called the "Hönninger Sande". The "Hönninger Sande" are correlated with the "Mosbacher Sande", namely with the middle and the upper part of the "Mittlere" respectively "Grauen Mosbachs" near Wiesbaden. As a result of this correlation the older main terrace in the Lower Middle Rhine Valley is classified as part of the Cromer Complex.

In this paper a more differentiated subdivision of these sand deposits is suggested. Only the lower part of the different profiles should be determinated as the fluvial "Hönninger Sande" in a specific sense. The thick upper parts of the sand deposits however consist of reworked sediments amongothers the "Hönninger Sande". They are then called "Linzer Sande". This redeposition took place by fluvial and eolian transport and solifluction. Consequently most of these sand deposits in the level of the older main terrace are of no stratigraphic value.

The statistic evaluation was the result of moment measures. Standard deviation and the mean are then very useful statisical tools for the characterization of a sediment. The grain size analysis showed that these good to excellent sorted sands require a sieve distance of 0,125 Zeta to come up to the Gauß rules for the plotting of the normal distribution in a log probability scale.

\section{Einleitung}

Das Gebiet des unteren Mittelrheins (Abb. 1) ist seit über 150 Jahren Arbeitsgebiet der Eiszeitenforschung. Im Bereich der Terrassenstratigraphie wurde von vielen Autoren eine Unterteilung vorgeschlagen (u. a. POHLIG 1883; JUNGBLUTH 1918; KAISER 1961; BIBUS 1980). In Tabelle 1 ist die Stratigraphie am unteren Mittelrhein nach verschiedenen Autoren dargestellt. Eine Zusammenfassung zur Korrelation mit anderen Abschnitten des Rheins befindet sich bei BOENIGK (1990). Die größten Unsicherheiten für eine stratigraphische Gliederung bestehen im Altquartär, den Terrassen, die älter sind als die Mittelterrassen. 
Tabelle 1: Stratigraphie am unteren Mittelrhein nach verschiedenen Autoren.

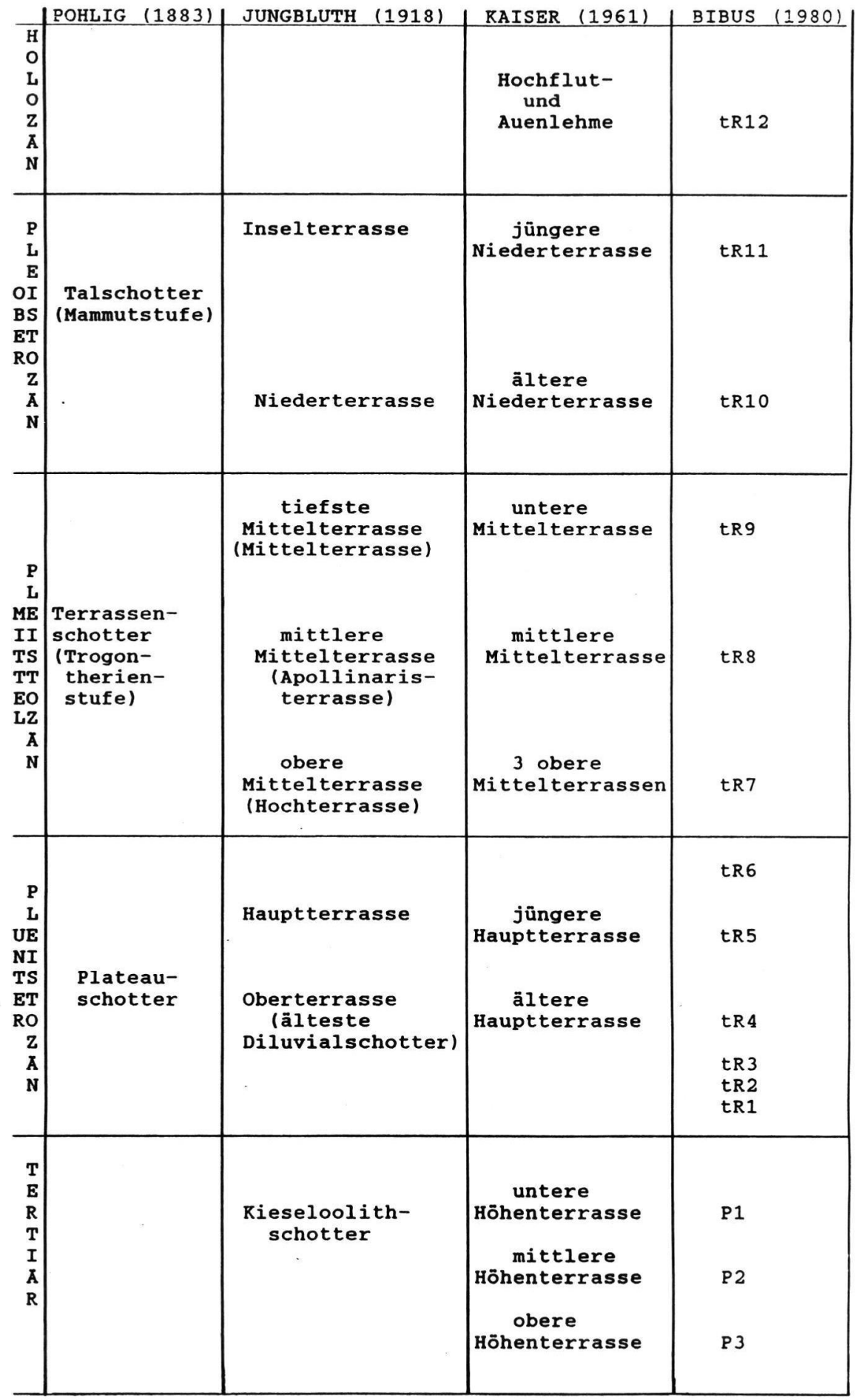




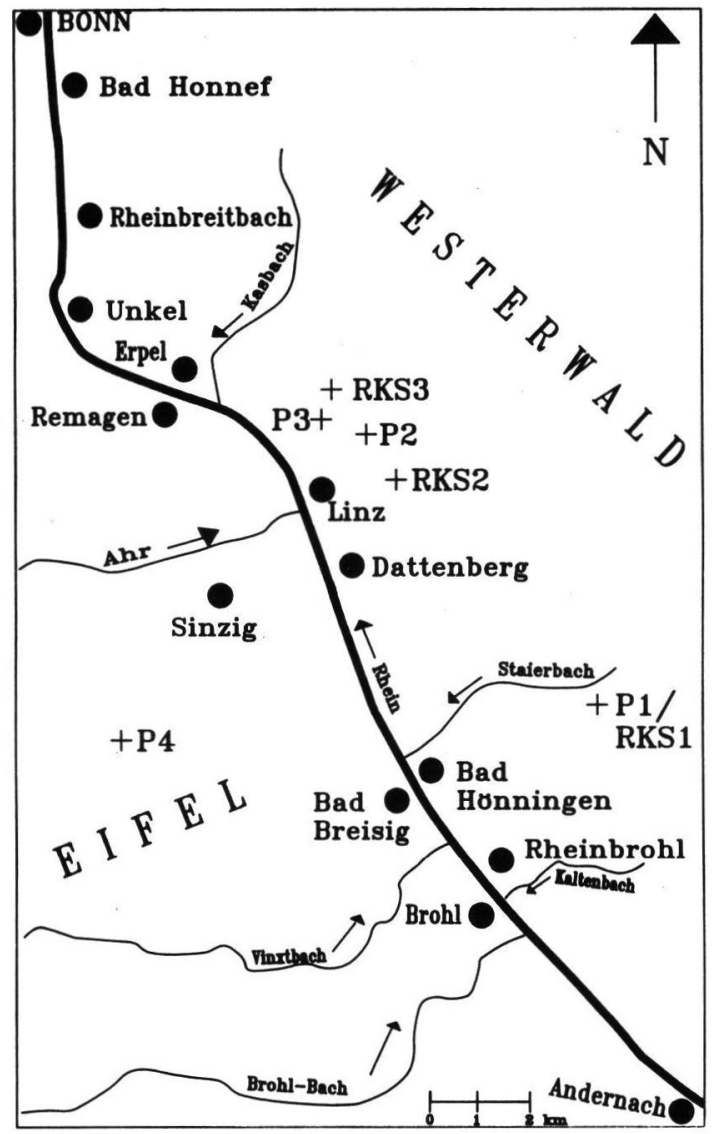

Abb. 1: Übersichtskarte des Arbeitsgebietes mit Lage der bearbeiteten Profile;

$\mathrm{P}=$ Profil $; \mathrm{RKS}=$ Rammkernsondierung (siehe Anhang 8.1).
Ein Problem ist, daß komplette Deckschichtenabfolgen weitestgehend fehlen. Desweiteren ist die petrographische Varianz der einzelnen Terrassen nicht eindeutig. Somit ist nur eine deutliche Trennung von den tertiären Terrassen, aufgrund eines wesentlich höheren Quarzgeröllanteils und einer veränderten Schwermineralführung, möglich.

Einen ersten Ansatzpunkt zur stratigraphischen Einordnung der Hauptterrassen bietet das Profil in der Tongrube von Mülheim-Kärlich, am Rand des Neuwieder Beckens, da hier eine sehr differenzierte Deckschichtenabfolge vorhanden ist (Zusammenfassung bei SCHIRMER 1990).

Einen zweiten Arbeitsansatz bot dann die Beschreibung der $\mathrm{H}$ ö $\mathrm{n} n$ inger $\mathrm{S}$ a nd e von BIBus (1980). Hierbei handelt es sich um bis zu $30 \mathrm{~m}$ mächtige Fein- bis Mittelsande im Bereich der älteren Hauptterrasse (äHT). Diese korreliert BIBUS (1980: 109) nach makroskopischem Vergleich mit den $\mathrm{M}$ os b a cher S a nden und zwar mit dem mittleren und oberen Teil des „Mittleren” bzw. „Grauen” Mosbachs bei Wiesbaden. Die Mosbacher Sande sind detailliert untersucht (u. a. KINKELIN 1889, 1901; BRÜNING 1970, 1974, 1978; GEISSERT 1970; HeIM 1970; BOENIGK 1977/78). Der oben angegebene Abschnitt aus Mosbach wird aufgrund paläontologischer Befunde in den Cromer-Komplex i.w.S. gestellt (Zusammenfassung bei BRUNING (1978)).

Einen Überblick zur Terrassenstratigraphie am unteren Mittelrhein bietet Abbildung 2. Hier erfolgt die Einstufung im Wesentlichen nach morphologischen Kriterien.

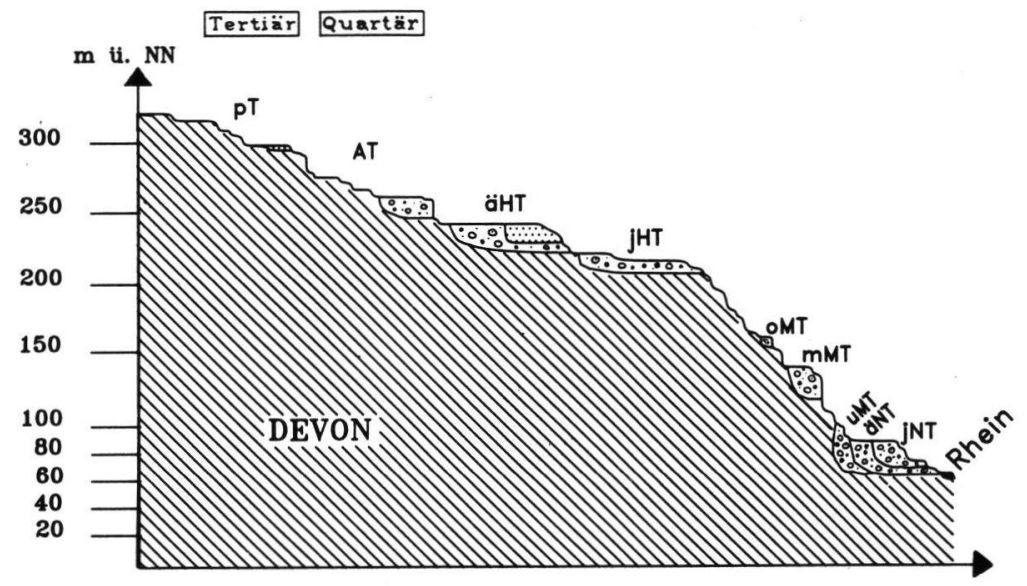

Abb. 2: Schematisches Terrassenprofil durch die Terrassentreppe am unteren Mittelrhein.

pT = pliozäne Terrassen; AT = Altpleistozäne Terrassen; äHT = ältere Hauptterrasse; $\mathrm{jHT}=$ jüngere Hauptterrasse; $\mathrm{oMT}=$ obere Mittelterrasse; $\mathrm{mMT}=$ mittlere Mittelterrasse; uMT = untere Mittelterrasse; äNT = ältere Niederterrasse; jNT = jüngere Niederterrasse. 


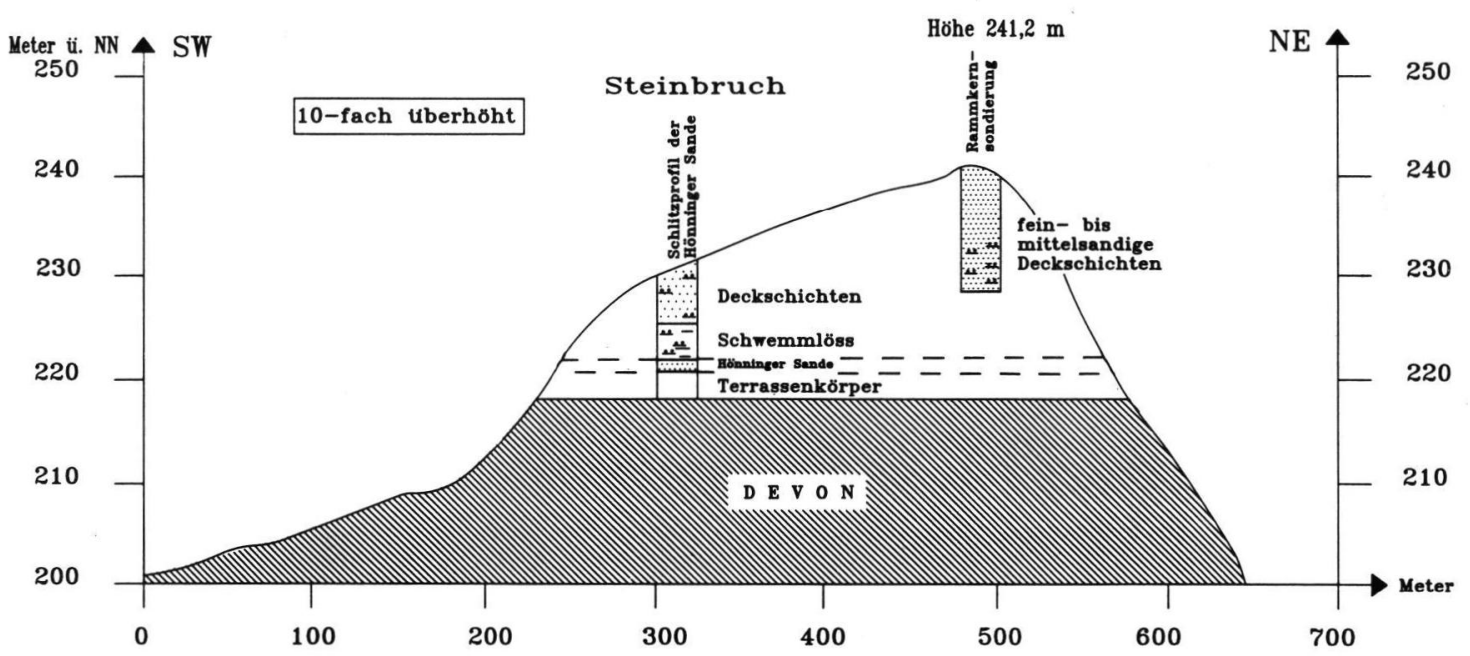

Abb. 3: Schematisches SW-NE Profil durch die Höhe 241,2 m und den Devonsteinbruch der Mannesmann AG mit seinen Deckschichten bei Bad Hönningen.

Die vorliegenden Untersuchungen gehen der Frage nach, ob alle Sandvorkommen im Bereich der äHT am unteren Mittelrhein, wie bei BIBUS (1980) angegeben, den Hönninger Sanden zugeordnet werden können.

\section{Geologische Untersuchungen}

Auf der Basis von Diplom-Kartierungen im Bereich des gesamten unteren Mittelrheins wurden vier Profile und drei Rammkernsondierungen ausgewählt und bearbeitet, die makroskopisch die Fazies der Hönninger Sande repräsentieren (Abb. 1 und Anhang).

Diese Profile wurden beschrieben und insgesamt 196 Proben genommen. Außerdem wurden noch Vergleichsproben der Mosbacher Sande aus den entsprechenden Profilabschnitten des Dyckerhoff-Steinbruchs bei Wiesbaden genommen. Die Proben wurden mit Hilfe der Korngrößenanalyse und der Schwermineralanalyse ausgewertet. Bei der vorliegenden Arbeit werden aus Platzgründen nur die Ergebnisse der Typlokalität der Hönninger Sande (Mannesmannaufschluß) östlich von Bad Hönningen (P1 und RKS1) und des Profils östlich von Linz (P2) dargestellt.

Bei den von BIBUS (1980) beschriebenen Hönninger Sanden handelt es sich um fluviatile Fein- bis Mittelsande. Sie sind olivgrünlich bis grau und weisen einen deutlichen Karbonatgehalt auf. Weiterhin ist ein deutlicher Glimmeranteil, mit Plättchen bis $5 \mathrm{~mm}$ Durchmesser, und eine schwarze Sprenkelung charakteristisch. Durch Karbonatumsatz kommt es zur Bil- dung von Kalksandsteinkonkretionen (Sandkindel). Von BIBUS (1980) wurden auch Schneckenbruchstücke ausgesiebt. Die Mächtigkeit der Sande im Bereich der Typlokalität beträgt mit dem auf dem Devon (unteres Siegen) aufliegenden ca. 1,88 m mächtigen Terrassenkörper $23,2 \mathrm{~m}$.

\section{Legende zu den Profilbeschreibungen}

Sedimentologie:

\begin{tabular}{|c|c|c|c|}
\hline$\because \because \because 0$ & $=$ Kies, kiesig & $\because \because \because$ & $=$ Mittelsand, mittelsandig \\
\hline $0_{0}^{0} \theta^{\circ}$ & = Grobkies, grobkiesig & $\because \square$ & $=$ Feinsand, feinsandig \\
\hline 000 & $=$ Mittelkies, mittelkiesig & 落 & $=$ Schluff, schluffig \\
\hline$\because \because \because: 0$ & $=$ Feinkies, feinkiesig & -二- & $=$ Ton, tonig \\
\hline$\because 6$ & $=$ Sand, sandig & 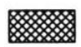 & $=$ Bodenbildung \\
\hline$\because \because \because::$ & = Grobsand, grobsandig & & \\
\hline
\end{tabular}

Schwermineralanalyse:

\begin{tabular}{|c|c|c|c|}
\hline Ella & $=$ Granat & 臂学 & $=$ Hornblende \\
\hline & $=$ Epidot & & $=$ Pyroxen \\
\hline & $=$ Alterit & & $=$ Rest \\
\hline
\end{tabular}

Abb. 4: Legende zu den Profilskizzen der Abb. 5-7. 


\subsection{Der Mannesmannaufschluß bei Bad Hönningen}

Dieser Aufschluß (P1, Abb. 1) wurde zuerst von BURRE (1928) beschrieben. Es handelt sich um den Aufschluß, der bei BiBUS (1980) die Typlokalität der Hönninger Sande darstellt. Es ist ein 11,35 m mächtiger Schurf. Auf dem höchsten Punkt der Erhebung $(241,2 \mathrm{~m}$ ü. NN), ca. $200 \mathrm{~m}$ vom Schurf entfernt, wurde eine Rammkernsondierung (RKS1) abgeteuft. Diese läßt sich mit dem Profil (P1) korrelieren (Abb. 3).

\subsubsection{Profilbeschreibung des Schurfs (P1)}

Die Abbildung 5 zeigt den Schichtaufbau und die Schwermineralverteilung des Profils, in dem etwa $11,35 \mathrm{~m}$ mächtige quartäre Ablagerungen folgender Zusammensetzung aufgeschlossenen sind.
Profilbeschreibung P1:

$m$ unter Profil-

oberkante

Zuord-

(Mächtigkeit der

Schicht in $\mathrm{m}$ )
Beschreibung

nung
$-1,9$

$(1,6)$

$-3,12$

$(1,22)$
$0,3 \quad$ humose Bodenauflage
$(0,3)$

Fein- bis Mittelsand, rötlich-braun, wechsellagernd mit Schluff, beigebraun, schwach glimmerhaltig, keine Schichtung

Feinsand, braun, schwach glimmerhaltig, stark verfestigt, Verwürfe, keine Schichtung

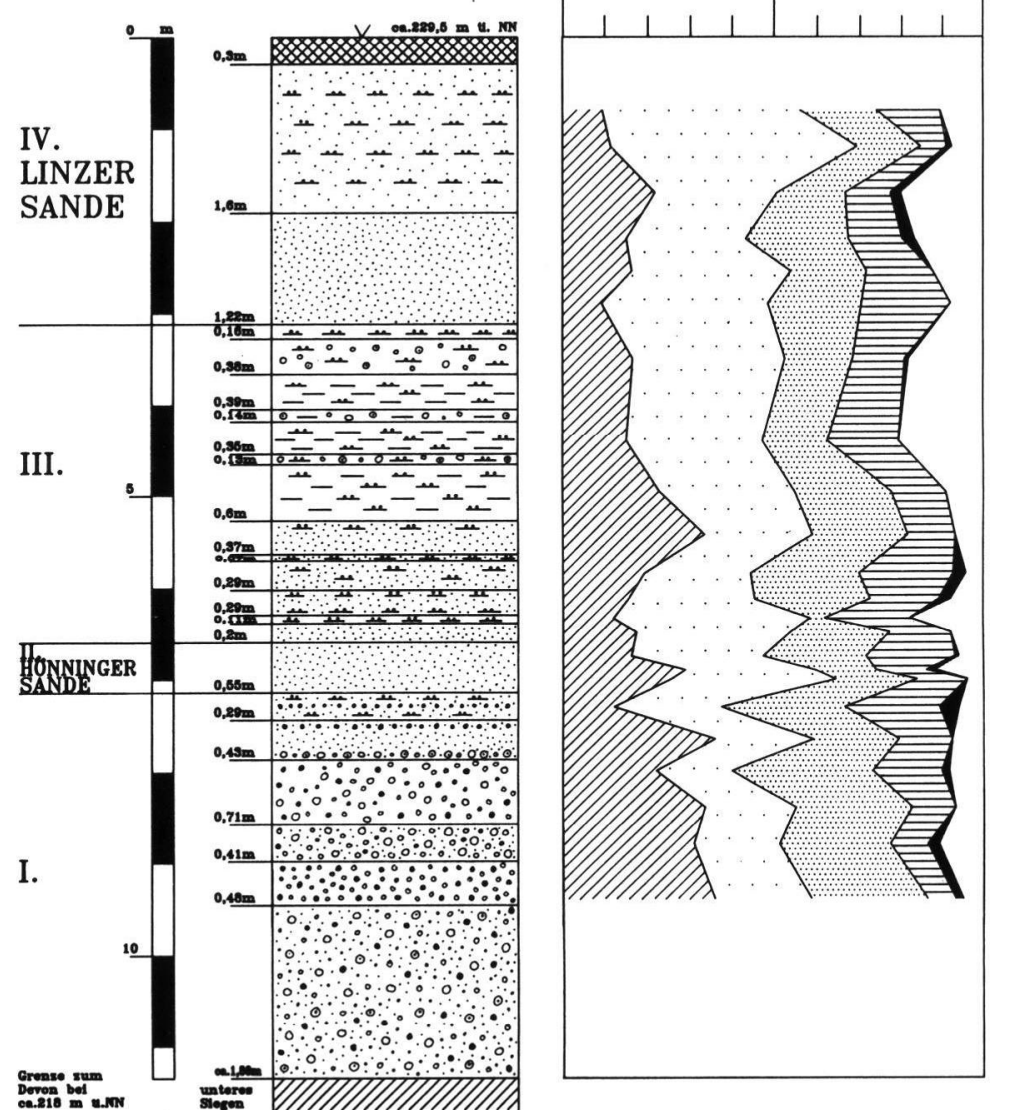

Abb. 5: Schematische Skizze und Schwermineraldiagramm des Schurfs P1 an der Typlokalität der Hönninger Sande im Mannesmann-Aufschluß östlich Bad Hönningen Legende siehe Abb. 4. 
Fortsetzung Profilbeschreibung P1:

m unter Profil- Beschreibung oberkante (Mächtigkeit der

Schicht in $\mathrm{m}$ )

$-3,28$

$(0,16)$

$-3,66$

$(0,38)$

$-4,05$

$(0,39)$

$-4,19$

$(0,14)$

$-4,54$

$(0,35)$

$-4,76$

$(0,13)$

$-5,27$

$(0,60)$

$-5,64$

$(0,37)$

$$
-5,71
$$

$-6,00$

$-6,29$

$(0,29)$

$-6,40$

$(0,11)$

$-6,60$

$(0,20)$
Schluff, hellgrau-gelb

III

Kies, schluffig, grau-braun,

Schuttband mit devonischen

Gesteinsbruchstücken,

Fe-Mn-hydroxid verkrustet

Schluff, tonig, beige-braun, mit Fe-hydroxidbändern

Schluff, stark kiesig, grau-braun, plattige und gerollte devonische

Gesteinsbruchstücke

Schluff, tonig, beige-braun,

Karbonatkonkretionen

Schluff, stark kiesig, grau-braun, plattige und gerollte devonische

Gesteinsbruchstücke

Schluff, tonig, beige-grau

Feinsand, nach oben schluffig, beige-braun, glimmerhaltig, karbonatisch, Lößkindel, keine Schichtung, Fe-hydroxidkruste bei $5,30 \mathrm{~m}$

Schluff, grün-grau, wechselgelagert mit Feinsand, beige-braun, karbonatische Konkretionen, Versätze an Störungen im cm-Bereich

Feinsand, schwach schluffig, beige-braun, glimmerhaltig

Schluff, rötlich-braun, wechselgelagert mit Feinsand, grün-grau

Schluff, gelblich-braun, grünliche Bänder, glimmerhaltig

Fein- bis Mittelsand, grünlichgrau, schwarz gesprenkelt, an Unterkante Sandkindel, glimmerhaltig, horizontal geschichtet

Fein- bis Mittelsand, grünlich-
Fortsetzung Profilbeschreibung P1:

Zuord- m unter Profil- Beschreibung

Zuord-

oberkante

nung

(Mächtigkeit der

Schicht in m)

$\begin{array}{cl}-7,44 & \text { Feinsand, schwach schluffig, } \\ (0,29) & \begin{array}{l}\text { dünne Grobsandbändchen, } \\ \text { rötlich-braun, karbonatisch }\end{array} \\ -7,87 & \begin{array}{l}\text { Feinsand, unten sehr schwach } \\ \text { kiesig, oben grobsandig, } \\ \text { gelblich-braun, undeutlich } \\ \text { geschichtet, Glimmer, karbonatisch }\end{array} \\ & \text { Mittel- bis Grobsand, fein- bis } \\ -8,58 & \text { mittelkiesig, nach oben hin Korn- } \\ (0,71) & \text { größenabnahme, gelblich-braun, } \\ & \text { oben schräggeschichtet und } \\ & \text { Mn-hydroxidbänder, karbonatisch } \\ -8,99 & \begin{array}{l}\text { Fein- bis Mittelkies, stark } \\ \text { sandig, gelblich-braun und } \\ \text { rötlich-schwarz, undeutlich } \\ \text { geschichtet, Mn-hydroxidbänder }\end{array} \\ & \begin{array}{l}\text { Grobsand, schwach feinkiesig, gelb- } \\ \text { lich-braun und rötlich-schwarz }\end{array} \\ -9,47 & \text { Terrassenkies, ergraben, Grenze } \\ (0,48) & \text { zum Devon erbohrt, Quarzgehalt } \\ \text { bei durchschnittlich 33\% }\end{array}$

Liegendes: kaolinitisch verwitterter Sandstein, Devon (Untere Siegener-Schichten)

$$
\begin{gathered}
\text { 2.1.2 Profilbeschreibung } \\
\text { der Rammkernsondierung (RKS1) }
\end{gathered}
$$

Die Abbildung 6 zeigt den Schichtaufbau und die Schwermineralverteilung der Rammkernsondierung an Punkt 241,2 m ü. NN.

Die Bohrung wurde $13 \mathrm{~m}$ tief abgeteuft, ohne daß Kies oder gar devonische Gesteine angetroffen wurden.

\section{Profilbeschreibung RKS1:}

$m$ unter Profil- Beschreibung

Zuordoberkante nung

(Mächtigkeit der

Schicht in $\mathrm{m}$ ) humose Bodenauflage 


\section{Schwermineralanalyse}

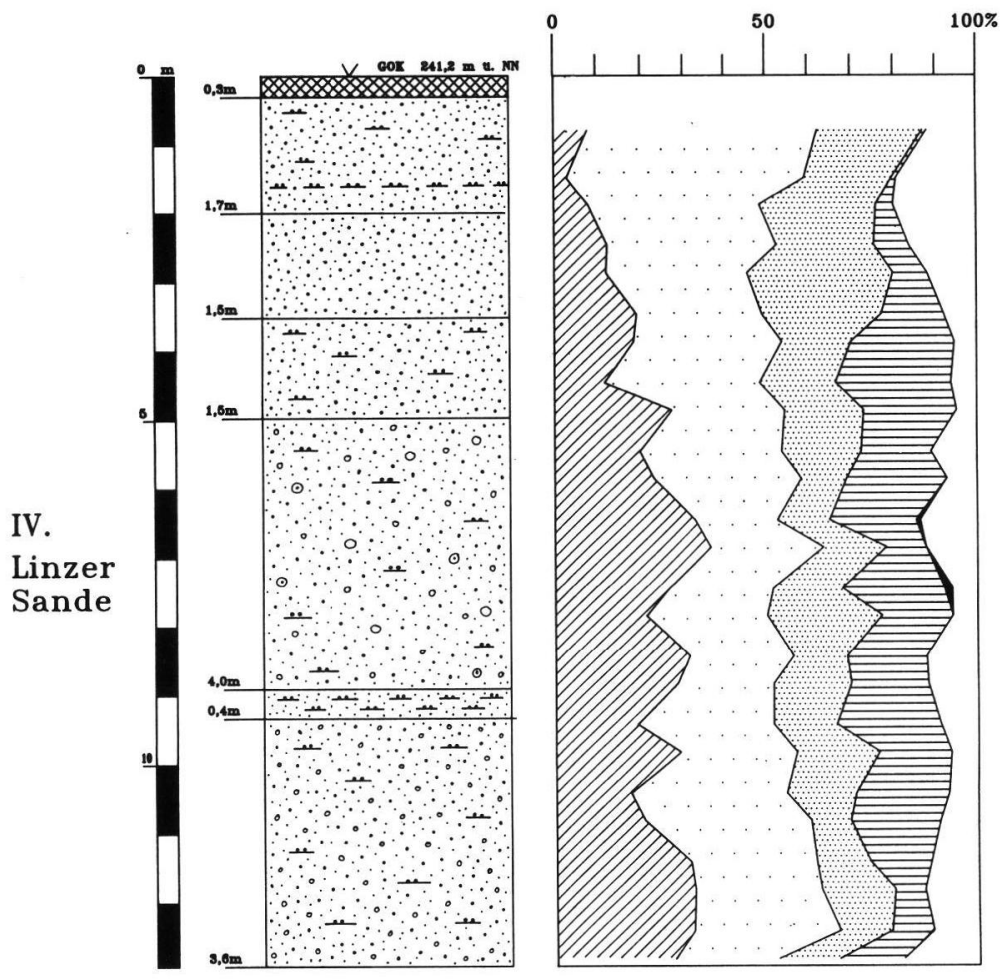

Abb. 6: Rammkernprofil (RKS 1) oberhalb der

Typlokalität der Hönninger Sande mit Diagramm der Schwermineralanalyse Legende siehe Abb. 4.

Schwermineralanalyse

Abb. 7: Schematische Skizze des Profils (P2) oberhalb von Linz mit Schwermineraldiagramm Legende siehe Abb. 4.

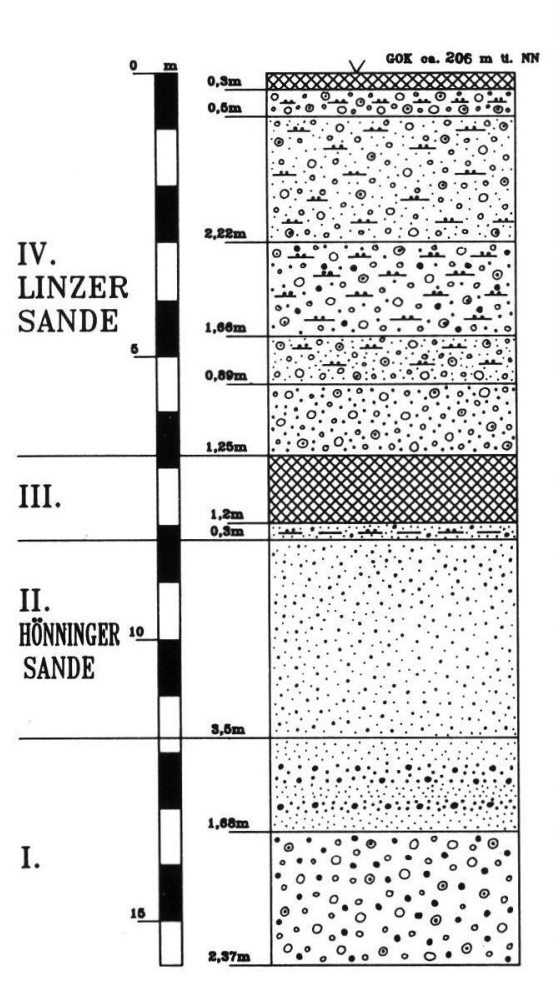


Fortsetzung Profilbeschreibung RKS 1:

$\begin{array}{lll}\text { m unter Profil- } & \text { Beschreibung } & \begin{array}{l}\text { Zuord- } \\ \text { nung }\end{array} \\ \text { oberkante } & \text { Mächtigkeit der } & \\ \text { Schicht in } \mathrm{m} \text { ) } & & \end{array}$

$\begin{aligned}-2,00 & \begin{array}{l}\text { Feinsand, mittelsandig, } \\ \text { rötlich-braun, glimmerhaltig, } \\ \text { Schluffbänder }\end{array} \\ -3,70) & \text { IV } \\ (1,50) & \begin{array}{l}\text { Feinsand, mittelsandig, } \\ \text { gelblich-braun bis rötlich-braun, } \\ \text { glimmerhaltig }\end{array} \\ -5,00 & \begin{array}{l}\text { Feinsand, mittelsandig, schwach } \\ \text { schluffig, gelblich-braun, Glimmer, }\end{array} \\ (1,50) & \text { karbonatisch } \\ -9,00 & \begin{array}{l}\text { Feinsand, mittelsandig, schwach } \\ \text { schluffig und kiesig, Glimmer, }\end{array} \\ (4,00) & \text { karbonatisch mit Konkretionen } \\ -9,40 & \begin{array}{l}\text { Schluff, schwach feinsandig, } \\ \text { gelblich-braun, karbonatisch }\end{array} \\ (0,40) & \text { Feinsand, schwach mittelsandig } \\ -13,00 & \text { und schluffig, sehr schwach fein- } \\ (3,60) & \text { sandig, glimmerhaltig, karbonatisch }\end{aligned}$

\subsection{Das Profil bei Linz}

Das Profil (P 2) oberhalb von Linz wurde zuerst von BIBUS (1980) beschrieben. Es befindet sich ca. $1 \mathrm{~km}$ östlich von Linz zwischen dem Waschberg und Gut Frühscheid. Der Aufschluß liegt in einem Gebiet, in dem früher Kiese der jüngeren Hauptterrasse abgebaut wurden. In diesem Bereich ist keine Geländestufe von der jHT zur äHT zu erkennen. Die Oberkante des Aufschlusses liegt bei ca. $206 \mathrm{~m}$ ü. NN, die Grenze zum Devon befindet sich bei ca. $185 \mathrm{~m}$ ü. NN. $\mathrm{Da}$ in diesem Gebiet bei normaler Lagerung die Unterkante der äHT bei ca. $215 \mathrm{~m}$ ü. NN liegt, muß die äHT hier bei Linz um ca. $30 \mathrm{~m}$ abgesenkt sein.

\subsubsection{Profilbeschreibung des Schurfs (P 2)}

Die Abbildung 7 zeigt den Schichtaufbau und die Schwermineralverteilung des Profils (P 2).

Aufgeschlossen sind etwa 15,87 m quartäre Sedimente. Durch eine Bohrung unmittelbar am Fuß des Schurfes wurden ca. $5 \mathrm{~m}$ Kies erschlossen.
Profilbeschreibung P2:

m unter Profil- Beschreibung

Zuordoberkante

(Mächtigkeit der

Schicht in $\mathrm{m}$ ) $\begin{array}{cc}-0,30 & \text { humose Bodenauflage } \\ (0,30) & \end{array}$

$-0,80 \quad$ Kies, schluffig, braun,

$(0,50) \quad$ devonischer Spülschutt

$-3,02$ Feinsand, stark kiesig, schluffig,

$(2,22)$ braun, rostfarben gestreift, devonische Gesteinsbruchstücke, keine Schichtung

\begin{tabular}{|c|c|}
\hline $\begin{array}{r}-4,68 \\
(1,66)\end{array}$ & $\begin{array}{l}\text { Mittel- bis Grobsand, stark } \\
\text { kiesig, schluffig, grau-braun, } \\
\text { Mollusken, devonische Gesteins- } \\
\text { bruchstücke, keine Schichtung }\end{array}$ \\
\hline $\begin{array}{r}-5,57 \\
(0,89)\end{array}$ & $\begin{array}{l}\text { Mittel- bis Grobsand, kiesig, } \\
\text { grau-braun, wechselgelagert } \\
\text { mit Feinsand, schluffig, kiesig, } \\
\text { grau und braun, fest, karbonatisc } \\
\text { Mollusken, devonische Gesteins- } \\
\text { bruchstücke }\end{array}$ \\
\hline $\begin{array}{r}-6,82 \\
(1,25)\end{array}$ & $\begin{array}{l}\text { Mittelsand, kiesig, grau-braun, } \\
\text { karbonatisch, Mollusken, } \\
\text { devonische Gesteinsbruchstücke, } \\
\text { keine Schichtung }\end{array}$ \\
\hline
\end{tabular}

$-8,02$

Schluff, tonig, schwach kiesig,

III braun, Bodensediment

$-8,32$

$(0,30)$

Schluff, tonig, sandig, gelblichbraun, stark karbonatisch, Mollusken

$-11,82$
$(3,50)$

$-\quad 13,50$
$(1,68)$

Fein- bis Mittelsande, grünlichII grau, schwarz gesprenkelt, viel Glimmer, gut sortiert, karbonatisch, kaum Schichtung

Feinsand, mittelgrau, viel I Glimmer, schwach karbonatisch, wechselgelagert mit Mittel- bis Grobsand, rötlich-grau zum Teil durch Fe-hydroxide rötlich-braun, zum Teil schräggeschichtet

$-15,87$ Grobsand, stark kiesig, $(2,37)$ rötlich-braun (Terrassenkörper)

Liegendes: Grenze nicht aufgeschlossen Devon (Mittlere Siegener-Schichten) 


\section{Geologische Interpretationen}

\subsection{Geologisch-petrographische Interpretationen}

Aufgrund der Bearbeitung der Schürfe und Rammkernsondierungen konnte eine charakteristische Vierteilung der Sandfazies im Bereich der äHT erarbeitet werden:

I. Terrassenkörper auf dem Devon der unteren Siegener-Schichten auflagernd, dessen Feinbis Mittelsandanteil karbonatisch ist; darüber folgt ein Übergangsbereich aus kiesigen Sanden. Es folgt:

II. Karbonatische Fein- bis Mittelsande = „HÖNNINGER SANDE” im engeren Sinne mit einer maximalen Mächtigkeit von $3,5 \mathrm{~m}$.

III. Schwemmschuttbereich aus Lößlehm mit Sand und devonischen Gesteinsbruchstücken; im Profil bei Linz mit Bodensediment.

IV. Bis über $20 \mathrm{~m}$ mächtige Deckschichtenabfolge aus Fein- bis Mittelsanden mit stärkerem Schluffanteil als II, devonischen Gesteinsbruchstücken und teilweise starker Molluskenführung; diese Sande werden in dieser Arbeit aufgrund ihrer charakteristischen Ausprägung im Profil bei Linz als „LINZER SANDE” bezeichnet.

Die Hönninger Sande i.e. S. sind gut sortierte fluviatile Sande, die als Abschluß der Schottersedimentation auftreten. Durch einen Anteil an Löß und zahlreichen devonischen Gesteinsbruchstücke geben sich die Linzer Sande dagegen als Umlagerungsprodukte zu erkennen, mit einem hohen Anteil an „Hönninger Sanden" im engeren Sinne. Für Linzer Sande ist außerdem charakteristisch, daß die Glimmerplättchen zerbrochen sind und häufig Schalenbruchstücke von Mollusken vorkommen. Unter dem Binokular zeigte sich desweiteren, daß der Karbonatgehalt bei den Hönninger Sanden i.e.S. primär ist und aus klaren Kristallbruchstücken im Sandbereich besteht, während er bei den Linzer Sanden durch Aggregate in der Schluffraktion und Überzüge auf Quarzkörner hervorgerufen wird.

\subsection{Schwermineralanalyse}

An drei Profilen, Bad Hönningen Steinbruch (P1), Bad Hönningen Rammkernsondierung (RKS 1) und Linz (P2), wurden Schwermineralanalysen durchgeführt.

Es wurde die Fraktion $0,4-0,06 \mathrm{~mm}$ Durchmesser bearbeitet. Die weite Fraktion wurde gewählt, da die
Sedimente Kies bis Schluff umfassen und damit eine sehr große Schwankung des mittleren Korndurchmessers zeigen. Die Proben wurden gesiebt, mit heißer konz. $\mathrm{HCl}$ gereinigt, mit $\mathrm{Na}$-Polywolframat in der Zentrifuge abgetrennt und die Schwerefraktion in Mountex eingebettet. Entlang von Linien wurden 100 transparente Schwerminerale bestimmt und ausgezählt. Die opaken Mineralkörner wurden als relative Häufigkeiten im Vergleich zu den transparenten Körnern gezählt.

In allen drei Profilen tritt ein sehr ähnliches Schwermineralspektrum auf. Dominant sind die Minerale Epidot, Granat, Alterit und grüne Hornblende. Daneben sind regelmäßig in geringen Anteilen stabile (Turmalin, Zirkon, Rutil) und seltener metamorphe (Staurolith, Andalusit, Sillimanit) Minerale anwesend. Diese Minerale erreichen zusammen bis maximal $20 \%$ der transparenten Körner. Hier hebt sich das Profil Linz insofern heraus, als in Linz durchgängig geringere Anteile als $10 \%$ dieser Mineralgruppe zu verzeichnen sind. Sporadisch treten in den Profilen Glaukophan, Spinell und Chloritoid auf. Charakteristisch für die untersuchten Sedimente ist ein regelmäßiges Auftreten, wenn auch in geringen Prozentsätzen, von stark angelösten Klinopyroxenen. Titanit kommt relativ häufig vor.

Die Analysenergebnisse stimmen im Wesentlichen mit den Ergebnissen von SCHEER (in BIBUS 1980) aus Bad Hönningen überein. SCHEER hat lediglich die Gruppe Alterit nicht ausgegliedert. Damit verschiebt sich die relative Häufigkeit der einzelnen Minerale etwas zu höheren Prozentsätzen.

Die Schwankungen der relativen Anteile der Minerale innerhalb der Profile ist auf Korngrößeneffekte zurückzuführen. Lediglich in der RKS1 Bad Hönningen läßt sich eine starke Verwitterungsauslese von der Oberfläche her erkennen. Der Pyroxen verschwindet, während Granat und Hornblende mit Annäherung an die Oberfläche zunehmend reduziert werden, was ein relatives Anwachsen von Epidot, Alterit und stabilen Mineralen zur Folge hat. Dieser Verwitterungseinfluß greift etwa $5 \mathrm{~m}$ tief in das Profil hinein und folgt der Entkalkung der Sedimente. Die Profile Bad Hönningen Steinbruch und Linz zeigen diese Verwitterungserscheinung nicht, was auf eine, erosionsbedingte, nicht so alte Oberfläche schließen läßt.

Die gleichmäßige Schwermineralführung der bearbeiteten Sedimente, von der Basis bis zum Top, gibt keinen Anlaß, die Schotter von den Hönninger Sanden i.e.S. und diese von den Linzer Sanden zeitlich in ihrer Entstehung zu trennen. Es handelt sich um pleistozäne Sedimente, wie die Vormacht der „RheinMinerale" bezeugt. Da zumindest die Hönninger 
Profile im Bereich des quartären Osteifelvulkanismus liegen, kann aus dem generell geringen Anteil an vulkanischen Schwermineralen auf ein Alter größer als das der Mittelterrassen geschlossen werden (RAZI RAD 1976; BRUNNACKER et al. 1978). Das heißt auch die Linzer Sande, die Umlagerungsprodukte aus den älteren Sedimenten darstellen, sind in den untersuchten Profilen, älter als die Mittelterrassen.

Schwierig wird die Frage nach der Bedeutung der geringen vulkanischen Komponente in der Schwermineralfraktion aller untersuchten Sedimente. Eine geringe Pyroxen-Führung setzt nach VINKEN (1959), FRECHEN
\& Heide (1969), MUSA (1974), SCHNƯtGEN et al. (1975), BOENIGK (1978) in der jüngeren Hauptterrasse bzw. Hauptterrasse 3 ein. Auch die von FRECHEN \& HeIDE (1969) in die Ältere Hauptterrasse gestellten Vorkommen gehören in die jüngere Hauptterrasse.

Nach morphologischen Befunden soll die gesamte hier behandelte Abfolge aber älter als die jüngere Hauptterrasse sein (BIBUS 1980). Es bleibt nur die Überlegung, daß die Pyroxene in den normalerweise karbonatfreien Sedimenten des Altpleistozäns aufgelöst werden und nur in den karbonatischen Ablagerungen, wie sie hier vorliegen, erhalten geblieben sind.

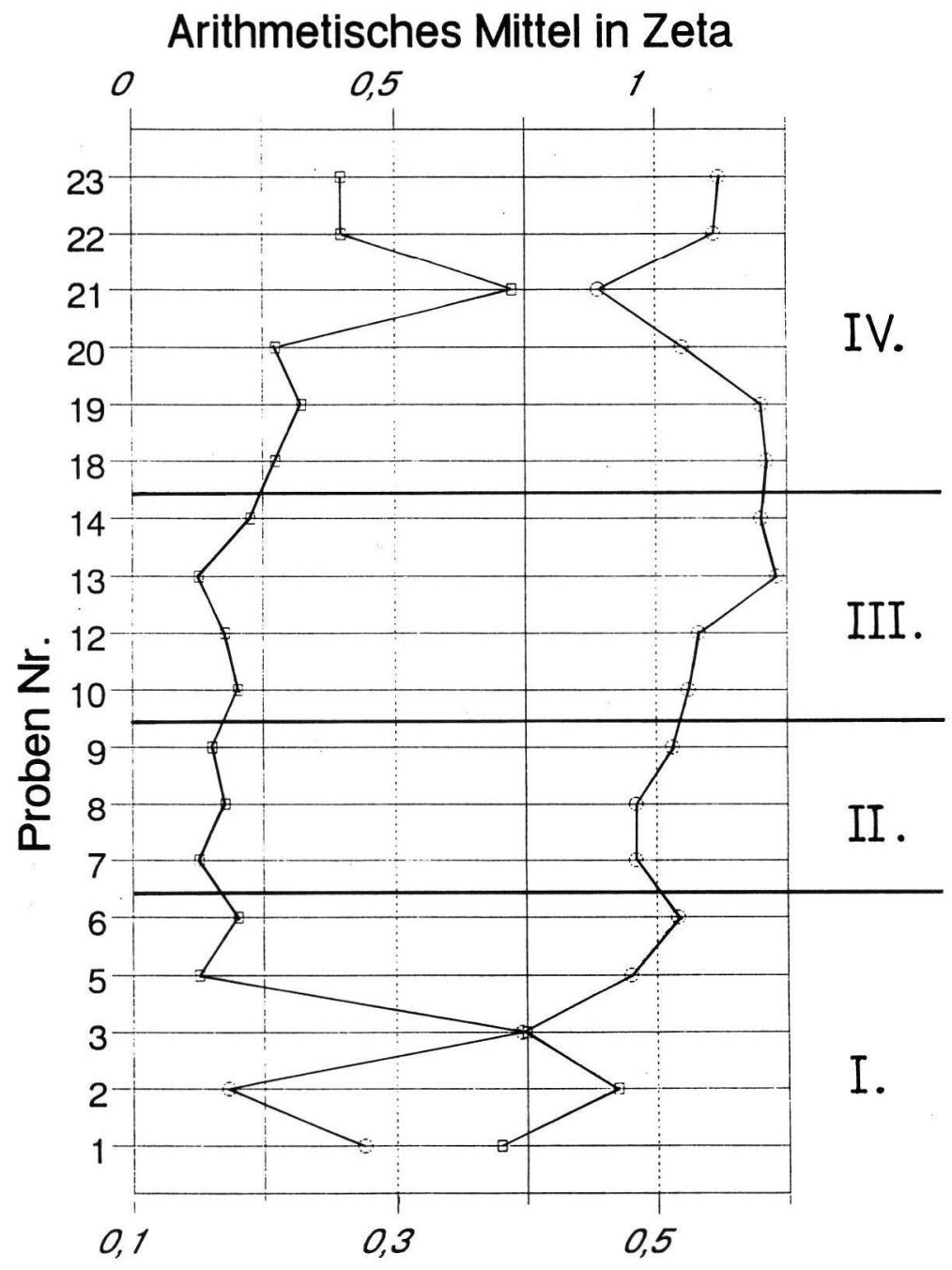

Standardabweichung in Zeta

= Arithmetisches Mittel

- = Standardabweichung

Abb. 8: Standardabweichung und arithmetisches Mittel an Proben der Typlokalität der Hönninger Sande (P1). 


\section{Statistik}

Es wurden alle 196 Proben gesiebt und diese mit Hilfe statistischer Methoden ausgewertet.

\subsection{Methoden}

Die Korngrößemanalysen wurden mit einem ZetaSiebsatz im 0,25 Zeta-Abstand (DIN 4022) durchgeführt.

Nach FÜCHTBAUER (1988) geht eine mathematisch einwandfreie und vollständige Charakterisierung von
Korngrößenanalysen von der Berechnung der Momente aus. Zur Berechnung der Momente wurden die Formeln nach MARSAL (1967) verwendet:

1. Moment: Arithmetisches Mittel (mean)

$$
\overline{\mathrm{X}}=\left(\mathrm{q}_{1} \mathrm{x}_{1}+\ldots+\mathrm{q}_{\mathrm{n}} \mathrm{x}_{\mathrm{n}}\right) / 100
$$

2. Moment: Standardabweichung

(standard deviation)

$\sigma=\left(\mathrm{q}_{1}\left(\mathrm{x}_{1}-\overline{\mathrm{X}}\right)^{2}+\ldots+\mathrm{q}_{\mathrm{n}}\left(\mathrm{x}_{\mathrm{n}}-\overline{\mathrm{X}}\right)^{2}\right.$

$(100)^{0} \cdot{ }^{5}\left(\right.$ Varianz $\left.=\sigma^{2}\right)$

$\mathbf{x}=$ Mittelpunkt der Kornfraktion in Zeta-Graden

$q=$ prozentuale Häufigkeit der Fraktion

\section{Arithmetisches Mittel in Zeta}

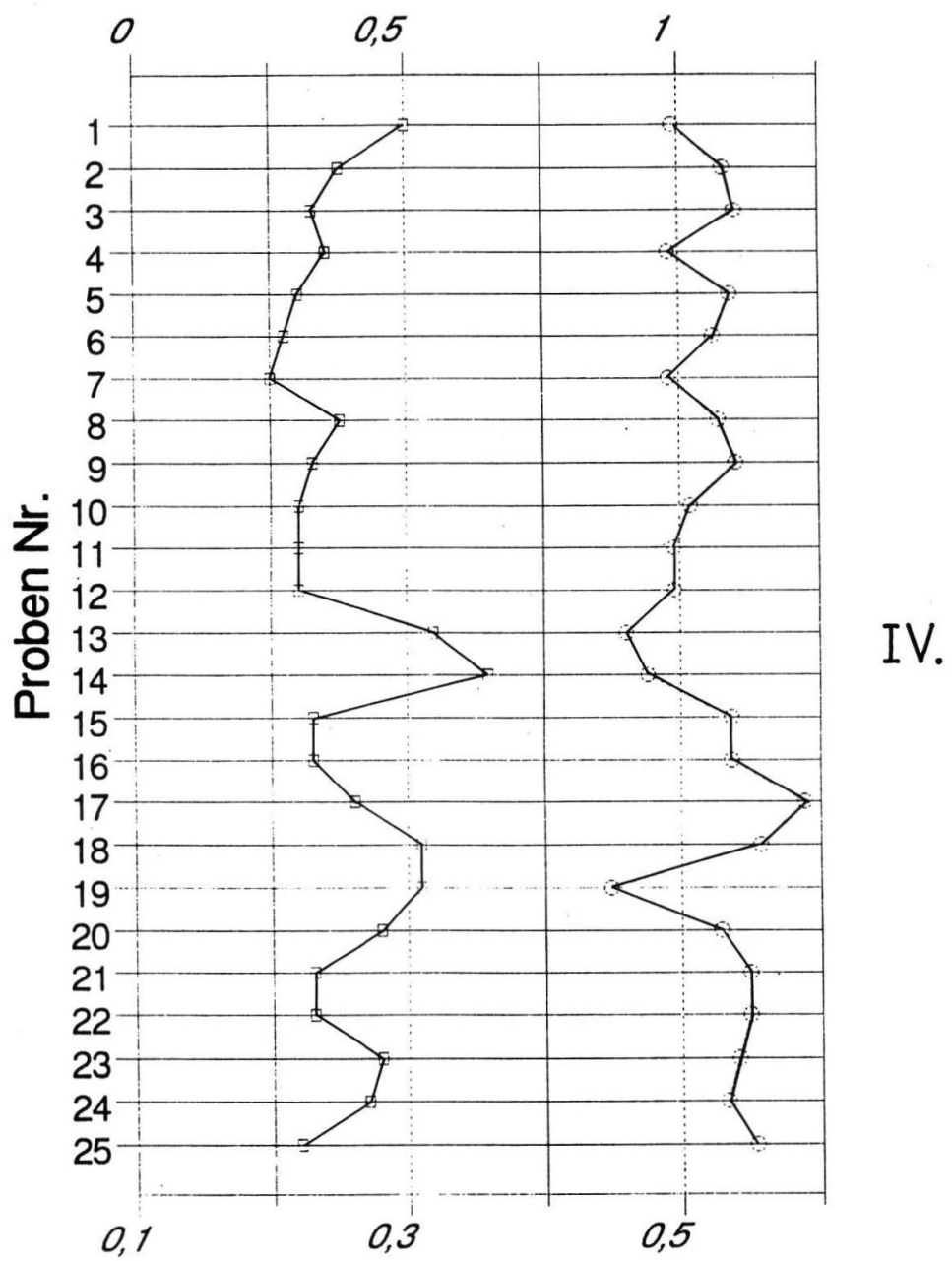

\section{Standardabweichung in Zeta}

= Arithmetisches Mittel

- = Standardabweichung
Abb. 9: Standardabweichung und arithmetisches Mittel an Proben der Rammkernsondierung oberhalb der Typlokalität (RKS1). 
3. Moment: Momentkoeffizient der Schiefe (skewness) $\alpha_{3}=\left(\mathrm{q}_{1}\left(\mathrm{x}_{1}-\overline{\mathrm{X}}\right)^{3}+\ldots+\mathrm{q}_{\mathrm{n}}\left(\mathrm{x}_{\mathrm{n}}-\overline{\mathrm{X}}\right)^{3}\right)$
$/ 100 * \sigma^{3}$

4. Moment: Momentkoeffizient der Steilheit (kurtosis)

$\alpha_{4}=\left(\mathrm{q}_{1}\left(\mathrm{x}_{1}-\overline{\mathrm{X}}\right)^{4}+\ldots+\mathrm{q}_{\mathrm{n}}\left(\mathrm{x}_{\mathrm{n}}-\overline{\mathrm{X}}\right)^{4}\right)$ $/ 100 * \sigma^{4}$

$\mathrm{x}=$ Mittelpunkt der Kornfraktion in Zeta-Graden $q=$ prozentuale Häufigkeit der Fraktion

Bei dieser Berechnung wird zwingend vorausgesetzt, daß die Abstände der Kornklassen immer gleich sind.
Die Ergebnisse der Berechnung der Momente sind im Anhang dargestellt.

Durch die Auswertung der Korngrößenanalysen konnte die Geländeinterpretation der Sandvorkommen am unteren Mittelrhein bestätigt werden. Die Abbildungen 8, 9 und 10 zeigen für die Aufschlußprofile bzw. Rammkernsondierungen von Bad Hönningen und Linz (P1, RKS 1 und $\mathrm{P} 2$ ) den Zusammenhang zwischen geologischer Interpretation und statistischer Auswertung.

Es wurde das 2. Moment dargestellt, da nach FUCHTBAUER (1988) die Standardabweichung am vollstän-

\section{Arithmetisches Mittel in Zeta}

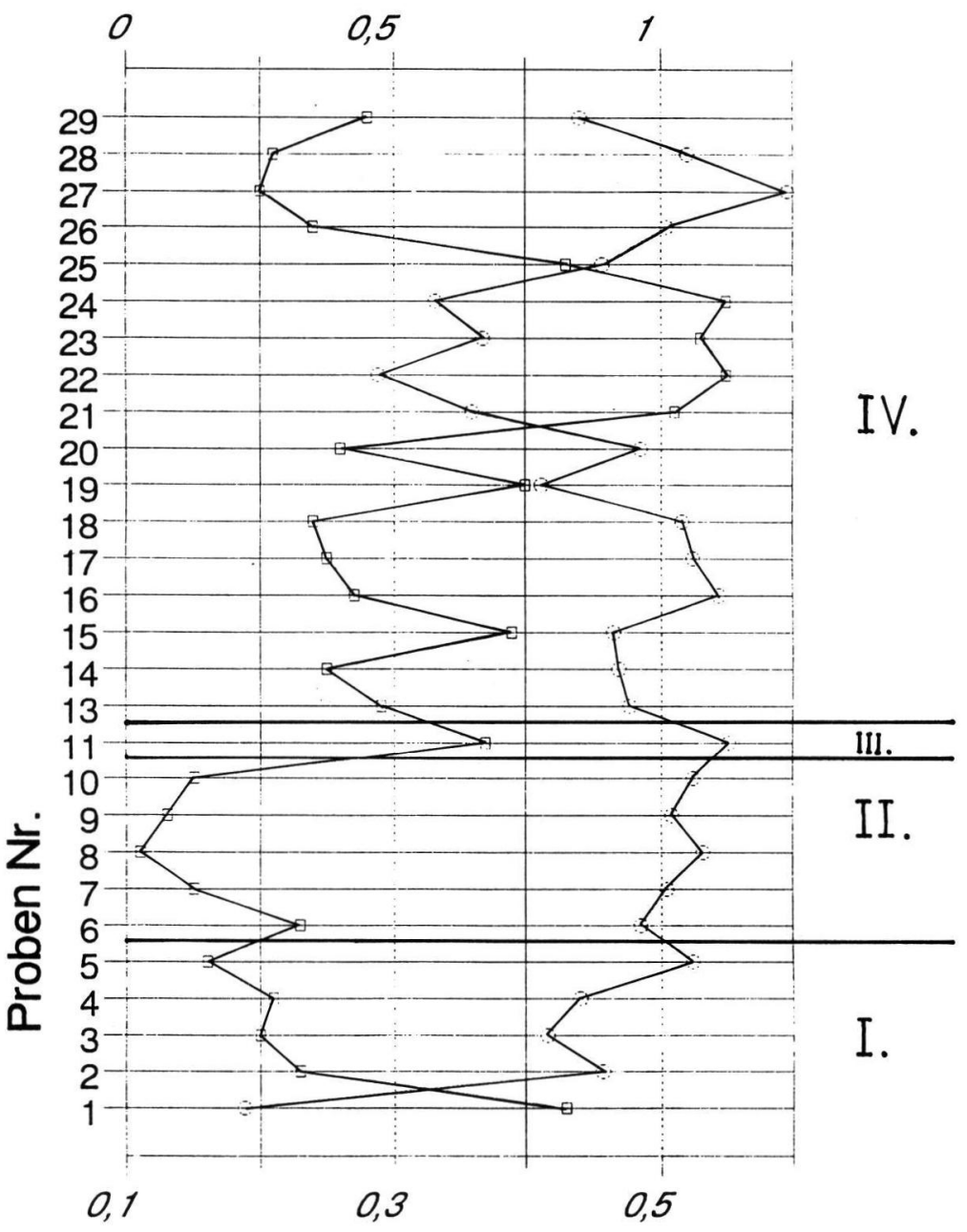

Standardabweichung in Zeta

= Arithmetisches Mittel

口 = Standardabweichung

Abb. 10: Standardabweichung und arithmetisches Mittel an Proben des Profils bei Linz - Typlokalität der Linzer Sande (P2). 
digsten die Sortierung ausdrückt. Desweiteren ist die Sortierung ein guter Indikator für das Ablagerungsmilieu eines Sedimentes. Zur weiteren Orientierung dient in den Abbildungen das 1. Moment (arithmetisches Mittel), welches eine leichte Korngrößenvergröberung zum Hangenden der Profile hin zeigt.

Vergleiche mit Proben der Mosbacher Sande ergaben, daß nur die unter II ausgegliederten Hönninger Sande mit dem mittleren bzw. grauen Mosbach, aufgrund der statistischen Parameter verglichen werden können. Beispiele für Korngrößenanalysen der Mosbacher Sande sind im Anhang dargestellt.

Das 3. und 4. Moment erbrachte für die Profile keine charakteristische Verteilung. Die vorhandenen Unterschiede können nicht auf klar definierte Veränderungen der Ablagerungsbedingungen zurückgeführt werden.

Bei der Überprüfung der Korngrößenanalysen auf Normalverteilung ergeben sich bei den gut sortierten Sanden häufig Anteile von $>50 \%$ in einer Klasse, da bei einem Klassenabstand von 0,25 Zeta nur $6 \mathrm{Klassen}$ im Sandbereich vorhanden sind. Die Anhäufung von mehr als $50 \%$ in einer Klasse ist aber nach den Gauß'schen Regeln zur Darstellung von Normalverteilungen im Wahrscheinlichkeitsnetz unzulässig; dies bedeutet, daß die Testverteilung eine kleinere Standardabweichung aufweist als die Gauß'sche Normalverteilung. Somit ist es bei so gut sortierten Proben, wie sie hier vorliegen, sinnvoll, die Klassenbreite zu halbieren $(0,125$ Zeta).

\section{Interpretation der Ergebnisse}

Bei den von BiBuS (1980) beschriebenen $\mathrm{H}$ ö n $\mathrm{n}$ i n g e r S a nde $\mathrm{n}$ handelt es sich um Fein- bis Mittelsande, die als stratigraphische Leitmarke verwendet werden. Durch Korngrößenanalyse und petrographische Beschreibung konnte aber eine deutliche Viergliederung für die Sandablagerungen im Bereich der älteren Hauptterrasse herausgearbeitet werden. Den $\mathrm{H}$ ön ninger $\mathrm{S}$ a nde $\mathrm{n}$ im engeren Sinne, Typ II, stehen die $\mathrm{L}$ in zer $\mathrm{S}$ a n d e, Typ IV gegenüber, die den größten Teil der Sandablagerungen ausmachen.

Die von BIBUS (1980: 109) vorgenommene Charakterisierung der Hönninger Sande treffen nur auf Typ II zu. Bei BiBus werden aber alle Sandvorkommen im Bereich der älteren Hauptterrasse als Hönninger Sande bezeichnet. Charakteristisch für die Linzer Sande sind dagegen eine schlechtere Sortierung als die Hönninger Sande i.e.S., gröbere arithmetisches Mittel und ein deutlicher Anteil an devonischen
Gesteinsbruchstücken, Löß und Lößlehm. Außerdem sind hier die Glimmerplättchen zerbrochen und es befinden sich in diesen Ablagerungen Schalenreste.

BIBUS (1980) bezeichnet die Feinsande insgesamt als fluviatile Sedimente, wobei die Ablagerung unter ausgeglichenen und ruhigen Transportverhältnissen und ohne größere seitliche Schuttzufuhr erfolgt sein soll. Der Uferrand soll nur wenige Zehner Meter entfernt gelegen haben. Es ist aber nur schwer vorstellbar, daß auf diese Weise Sedimentmächtigkeiten von bis zu 23 m entstehen. Da z. B. an der Typlokalität eine schützende Lößschicht fehlt, muß man sogar von einer ursprünglich noch größeren Mächtigkeit ausgehen. Aufgrund der hier vorgelegten Untergliederung der Sandablagerungen durch sedimentologische und petrographische Untersuchungen, soll folgendes Entstehungsmodell diskutiert werden:

Zur Zeit der älteren Hauptterrasse kam es zur Ablagerung eines mehrere Meter mächtigen Terrassenkörpers, der in seinem Fein- bis Mittelsandanteil karbonatisch ist (I). Es folgte eine Übergangsphase, in der es zu einer Korngrößenabnahme kam. Daraufhin muß es im Bereich des unteren Mittelrheins zu einer Beckenbildung gekommen sein. Während dieser Zeit wurden bei ruhigen Ablagerungsverhältnissen sehr gut sortierte Fein- bis Mittelsande abgelagert (Hönninger Sande i.e.S.). Zur Zeit der älteren Hauptterrasse hatte der Rhein im Raum Bad Hönningen noch eine Flußbreite von bis zu $8 \mathrm{~km}$, so daß die Sande weit verbreitet waren. Es erfolgte eine Sedimentationspause und eine Ablagerung von Schluff (Löß) (III). Nach dieser Ablagerung ist der Bereich der älteren Hauptterrasse bis heute um ca. $150 \mathrm{~m}$ gehoben worden, so daß langanhaltende Umlagerungsprozesse die Folge waren. Bei diesen Umlagerungen muß es sich um eine Kombination aus mehreren genetischen Prozessen handeln. Dabei kann man von Solifluktion, sowie von Verspülungen und äolischem Transport ausgehen. Die bis zu $20 \mathrm{~m}$ mächtigen Fein- bis Mittelsande, die sogenannten $\mathrm{L}$ i n z e r S a n d e (IV), sind also als Umlagerungsprodukte $u$. a. aus den Hönninger Sanden zu verstehen und können nicht als stratigraphischer Leithorizont dienen. Auch kann die Oberfläche nicht als Terrasse im Sinne einer fluviatilen Verebnungsfläche aufgefaßt werden.

Ähnliche Sande sind natürlich nicht auf den Bereich der älteren Hauptterrasse beschränkt sondern sind auch in jüngeren Terrassen zu finden. Hier wäre die Mittelterrasse von Ariendorf zu nennen. Dort befinden sich die „Linzer Sande” unterhalb des Lößkindelhorizontes des Kärlicher Interglazials in den Deckschichten.

Das bedeutet wiederum, daß jedes Sandvorkommen im Hauptterrassenbereich intensiv untersucht werden 
muß, um eine Zuordnung nach der Einteilung I-IV zu treffen. Es können theoretisch auch im Hauptterrassenbereich jüngere Sande auftreten.

\section{Danksagung}

Wir danken der DFG für die Unterstützung der Arbeiten.

\section{Schriftenverzeichnis}

Bibus, E. (1980): Zur Relief-, Boden- und Sedimententwicklung am unteren Mittelrhein. - Frankfurter Geow. Arbeiten, Serie D, 1: 296 S., Frankfurt a. M.

BoENIGK, W. (1977/78): Zur petrographischen Gliederung der Mosbacher Sande im Dyckerhoff-Steinbruch, Wiesbaden/Hessen. - Mz. Naturw. Arch., 16: 91-126; Mainz.

- (1978): Gliederung der altquartären Ablagerungen in der Niederrheinischen Bucht. - Fortschr. Geol. RheinId. u. Westf., 28: 135-212; Krefeld.

- (1990): Die pleistozänen Rheinterrassen und deren Bedeutung für die Gliederung des Eiszeitalters in Mitteleuropa. - In: LIEDTKE, H. [Hrsg.]: Eiszeitforschung, 130-140; Darmstadt (Wiss. Buchges.).

BRUNING, H. (1970): Zur Klima-Stratigraphie der pleistozänen Mosbacher Sande bei Wiesbaden (Hessen). Mz. Naturw. Arch., 9: 204-265; Mainz.

- (1974): Das Quartär-Profil im Dyckerhoff-Steinbruch, Wiesbaden/Hessen. - In: SeMMEL, A. [Hrsg.]: Das Eiszeitalter im Rhein-Main-Gebiet. - Rhein-Main-Forsch., 82: 57-81; Frankfurt a. M.

- (1978): Zur Untergliederung der Mosbacher-Terrassenabfolge und zum klimatischen Stellenwert der Mosbacher Tierwelt im Rahmen des Cromer-Komplexes. Mz. Naturw. Arch., 16: 143-190; Mainz.

Brunnacker, K., Boenigk, W., Dolezalek, B., Kempf, E. K., KoČI, A., MENTZEN, H., RAZI RAD, M. \& WINTER, K.-P. (1978): Die Mittelterrassen am Niederrhein zwischen Köln und Mönchengladbach. - Fortschr. Geol. Rheinld. u. Westf., 28: 277-324; Krefeld.

BURRE, O. (1928): Die älteste Diluvialterrasse bei Hönningen. - Jb. preuß. geol. L.-Amt, 49: 320-326; Berlin.

FreCHEN, J. \& HeIDE, H. (1969): Tephrostratigraphische Zusammenhänge zwischen der Vulkantätigkeit im Laacher See-Gebiet und der Mineralführung der Terrassenschotter am unteren Mittelrhein. - Decheniana, 122: 35-74; Krefeld.

FUCHTBAUER, H. [Hrsg.] (1988): Sedimente und Sedimentgesteine; Sediment-Petrologie II: 1141 S.; Stuttgart (Schweizerbart'sche).

GeISSERT, F. (1970): Mollusken aus den pleistozänen Mosbacher Sanden bei Wiesbaden (Hessen). - Mz. Naturw. Arch., 9: 147-203; Mainz.
Heim, D. (1970): Zur Petrographie der Mosbacher Sande. - Mz. Naturw. Arch., 9: 83-117; Mainz.

Jungbluth, F. A. (1918): Die Terrassen des Rheins von Andernach bis Bonn. - Verh. naturhist. Ver. preuß. Rheinld. u. Westf., 73: 1-103; Bonn.

KAISER, K. (1961): Gliederung und Formenschatz des Pliozäns und Quartärs am Mittel- und Niederrhein sowie in den angrenzenden Niederlanden unter besonderer Berücksichtigung der Rheinterrassen. - Festschr. 33 Dt. Geogr.-Tg. Köln: 236-278; Wiesbaden.

Kinkelin, F. (1889): Der Pliocänsee des Rhein- und Mainthales und die ehemaligen Mainläufe. - Ber. senckenb. naturforsch. Ges., 20: 39-150; Frankfurt a. M.

- (1901): Über das Vorkommen eines erratischen Blockes von Nummulitenkalk in den Mosbacher Sanden. - Z Z Deutsch. Geol. Ges., 53: 41-42; Berlin.

MARSAL, D. (1967): Statistische Methoden für Erdwissenschaftler. - Schweizerbart'sche Verlagsbuchhandlung: 152 S.; Stuttgart.

MUSA, J. (1974): Rhein- und Eifelschüttungen im Süden der Niederrheinischen Bucht. - Sonderveröffentl. Geol. Inst. Köln, 23: 151 S.; Köln.

PoHLIG, H. (1883): Geologisch-paläontologische Untersuchungen in der Umgebung von Bonn. - Verh. naturhist. Ver. preuß. Rheinld. u. Westf., 40: 228-235; Bonn.

RAZI, RAD, M. (1976): Schwermineraluntersuchungen zur Quartärstratigraphie am Mittelrhein. - Sonderveröffent. Geol. Inst. Univ. Köln, 28: 164 S.; Köln.

SCHIRMER, W. (1990): Kärlich-Forschungsstand 1990. In: SCHIRMER, W. [Hrsg.]: Rheingeschichte zwischen Mosel und Maas. - DEUQUA-Führer, 1: 60-67; Hannover.

SCHNƯtgen, A., BoENIGK, W., BRUNNACKer, M., KoČ̃I, A. \& BRUNNACKER, K. (1975): Der Übergang der Hauptterrassenfolge zur Mittelterrassenfolge am Niederrhein. —Decheniana, 128: 67-86; Krefeld.

VINKEN, R. (1959): Sedimentpetrographische Untersuchungen der Rheinterrassen im östlichen Teil der Niederrheinischen Bucht. - Fortschr. Geol. Rheinld. Westf., 4: 127-170; Krefeld.

\section{Anhang}

\subsection{Aufschlußverzeichnis}

1. Schurf der Typlokalität der Hönninger Sande (Mannesmannaufschluß) bei Bad Hönningen; R 259526 H 559994; (P1).

2. Rammkernprofil oberhalb der Typlokalität der Hönninger Sande;

R 259531 H 560004; (RKS1). 
3. Aufschluß bei Linz, Typlokalität der Linzer Sande; R 259220 H 560460; (P 2).

4. Rammkernprofil am Roniger Hof; R 259310 H 560335; (RKS 2).

5. Aufschluß bei Kasbach-Ohlenberg; R 259050 H 560608; (P 3).

6. Rammkernsondierung bei Kasbach-Ohlenberg; R 259092 H 560734; (RKS 3).

7. Aufschluß am Sinzigkopf (einziges linksrheinisches Profil); R 258730 H 559919; (P4).

8. Mosbacher Sande im Dyckerhoff-Steinbruch, Bf. Wiesbaden-Ost.

\subsection{Analysenergebnisse der statistischen Parameter und Teufen der Probenentnahmepunkte}

8.2.1 Schurf der Typlokalität (P1)

\begin{tabular}{|c|c|c|c|c|c|c|}
\hline Nr. & $\overline{\mathrm{X}}$ & $\sigma$ & $\sigma^{2}$ & $\alpha_{3}$ & $\alpha_{4}$ & Teufe \\
\hline Probe 1 & 0,44 & 0,38 & 0,14 & $-0,63$ & 2,44 & $9,40 \mathrm{~m}$ \\
\hline Probe 2 & 0,18 & 0,47 & 0,22 & 0,13 & 1,62 & $8,80 \mathrm{~m}$ \\
\hline Probe 3 & 0,74 & 0,40 & 0,16 & $-1,38$ & 5,64 & $8,40 \mathrm{~m}$ \\
\hline Probe 5 & 0,95 & 0,15 & 0,02 & $-0,05$ & 9,60 & $7,65 \mathrm{~m}$ \\
\hline Probe 6 & 1,04 & 0,18 & 0,03 & $-0,26$ & 5,90 & $7,30 \mathrm{~m}$ \\
\hline Probe 7 & 0,96 & 0,15 & 0,02 & 0,09 & 6,41 & $7,00 \mathrm{~m}$ \\
\hline Probe 8 & 0,96 & 0,17 & 0,03 & 0,08 & 2,50 & $6,90 \mathrm{~m}$ \\
\hline Probe 9 & 1,03 & 0,16 & 0,03 & 0,38 & 5,56 & $6,75 \mathrm{~m}$ \\
\hline Probe 10 & 1,06 & 0,18 & 0,03 & 0,25 & 7,69 & $6,50 \mathrm{~m}$ \\
\hline Probe 12 & 1,08 & 0,17 & 0,03 & 0,89 & 8,75 & $6,35 \mathrm{~m}$ \\
\hline Probe 13 & 1,23 & 0,15 & 0,02 & 0,41 & 18,58 & $5,85 \mathrm{~m}$ \\
\hline Probe 14 & 1,20 & 0,19 & 0,04 & 0,36 & 9,49 & $5,45 \mathrm{~m}$ \\
\hline Probe 18 & 1,21 & 0,21 & 0,04 & $-1,05$ & 6,98 & $4,95 \mathrm{~m}$ \\
\hline Probe 19 & 1,20 & 0,23 & 0,05 & $-0,97$ & 5,67 & $2,55 \mathrm{~m}$ \\
\hline Probe 20 & 1,05 & 0,21 & 0,04 & 0,31 & 6,04 & $2,20 \mathrm{~m}$ \\
\hline Probe 21 & 0,89 & 0,39 & 0,16 & $-1,13$ & 5,95 & $1,70 \mathrm{~m}$ \\
\hline Probe 22 & 1,11 & 0,26 & 0,07 & $-0,38$ & 5,49 & $1,20 \mathrm{~m}$ \\
\hline Probe 23 & 1,12 & 0,26 & 0,07 & $-0,44$ & 5,18 & $0,80 \mathrm{~m}$ \\
\hline
\end{tabular}

\subsubsection{Rammkernsondierung oberhalb von P1 (RKS1)}

$\mathrm{Nr}$.
$\overline{\mathrm{x}}$
$\sigma^{2}$
$\alpha_{3}$
$\alpha_{4} \quad$ Teufe in $\mathrm{m}$

\begin{tabular}{lcccccr}
\hline Nr. & $\overline{\mathrm{X}}$ & $\boldsymbol{\sigma}$ & $\sigma^{2}$ & $\alpha_{3}$ & \multicolumn{1}{c}{$\alpha_{4}$} & Teufe in m \\
\hline Probe 10 & 1,02 & 0,22 & 0,05 & $-0,40$ & 6,35 & $5,40-5,60$ \\
Probe 11 & 0,99 & 0,22 & 0,05 & $-0,11$ & 4,92 & $5,80-6,00$ \\
Probe 12 & 0,99 & 0,22 & 0,05 & $-0,88$ & 7,75 & $6,40-6,60$ \\
Probe 13 & 0,90 & 0,32 & 0,10 & $-1,23$ & 6,11 & $6,80-7,00$ \\
Probe 14 & 0,94 & 0,36 & 0,13 & $-1,58$ & 6,31 & $7,40-7,60$ \\
Probe 15 & 1,09 & 0,23 & 0,05 & $-0,15$ & 5,71 & $7,80-8,00$ \\
Probe 16 & 1,09 & 0,23 & 0,05 & $-0,26$ & 5,90 & $8,40-8,60$ \\
Probe 17 & 1,22 & 0,26 & 0,07 & $-1,06$ & 7,16 & $8,80-9,00$ \\
Probe 18 & 1,14 & 0,31 & 0,09 & $-0,89$ & 5,91 & $9,40-9,60$ \\
Probe 19 & 0,87 & 0,31 & 0,10 & $-0,80$ & 4,94 & $9,80-10,00$ \\
Probe 20 & 1,07 & 0,28 & 0,08 & $-1,76$ & 7,69 & $10,40-10,60$ \\
Probe 21 & 1,12 & 0,23 & 0,05 & $-0,51$ & 5,44 & $10,80-11,00$ \\
Probe 22 & 1,12 & 0,23 & 0,05 & $-0,61$ & 5,99 & $11,40-11,60$ \\
Probe 23 & 1,10 & 0,28 & 0,08 & $-2,16$ & 12,92 & $11,80-12,00$ \\
Probe 24 & 1,08 & 0,27 & 0,07 & $-1,60$ & 5,88 & $12,40-12,60$ \\
Probe 25 & 1,13 & 0,22 & 0,05 & $-0,13$ & 5,81 & $12,80-13,00$
\end{tabular}

8.2.3 Profil bei Linz (P 2)

\begin{tabular}{lcccrrr}
\hline Nr. & $\overline{\mathrm{X}}$ & $\sigma$ & $\sigma^{2}$ & \multicolumn{1}{c}{$\alpha_{3}$} & $\alpha_{4}$ & Teufe \\
\hline Probe 1 & 0,22 & 0,43 & 0,18 & $-0,11$ & 2,08 & $14,70 \mathrm{~m}$ \\
Probe 2 & 0,89 & 0,23 & 0,05 & $-1,11$ & 4,86 & $13,30 \mathrm{~m}$ \\
Probe 3 & 0,79 & 0,20 & 0,04 & 0,07 & 3,86 & $12,85 \mathrm{~m}$ \\
Probe 4 & 0,85 & 0,21 & 0,05 & $-0,40$ & 3,68 & $12,45 \mathrm{~m}$ \\
Probe 5 & 1,06 & 0,16 & 0,02 & 0,40 & 5,57 & $12,00 \mathrm{~m}$ \\
Probe 6 & 0,96 & 0,23 & 0,05 & $-2,29$ & 10,15 & $11,70 \mathrm{~m}$ \\
Probe 7 & 1,01 & 0,15 & 0,02 & 0,49 & 9,28 & $10,95 \mathrm{~m}$ \\
Probe 8 & 1,08 & 0,11 & 0,01 & $-0,79$ & 7,99 & $10,15 \mathrm{~m}$ \\
Probe 9 & 1,02 & 0,13 & 0,02 & 0,48 & 4,65 & $9,40 \mathrm{~m}$ \\
Probe 10 & 1,06 & 0,15 & 0,02 & $-0,18$ & 9,99 & $8,60 \mathrm{~m}$ \\
Probe 11 & 1,13 & 0,37 & 0,14 & $-1,05$ & 4,89 & $8,00 \mathrm{~m}$ \\
Probe 13 & 0,94 & 0,29 & 0,09 & $-0,98$ & 6,28 & $6,65 \mathrm{~m}$ \\
Probe 14 & 0,92 & 0,25 & 0,06 & $-0,53$ & 3,27 & $6,20 \mathrm{~m}$ \\
Probe 15 & 0,91 & 0,39 & 0,15 & $-1,72$ & 4,16 & $5,80 \mathrm{~m}$ \\
Probe 16 & 1,11 & 0,27 & 0,07 & $-1,30$ & 8,78 & $5,45 \mathrm{~m}$ \\
Probe 17 & 1,06 & 0,25 & 0,06 & $-0,71$ & 5,15 & $5,15 \mathrm{~m}$ \\
Probe 18 & 1,04 & 0,24 & 0,06 & $-2,03$ & 12,68 & $4,85 \mathrm{~m}$ \\
Probe 19 & 0,78 & 0,40 & 0,16 & $-1,39$ & 3,92 & $4,45 \mathrm{~m}$ \\
Probe 20 & 0,96 & 0,26 & 0,07 & $-0,97$ & 5,72 & $4,15 \mathrm{~m}$ \\
Probe 21 & 0,65 & 0,51 & 0,26 & $-0,86$ & 2,41 & $3,80 \mathrm{~m}$ \\
Probe 22 & 0,47 & 0,55 & 0,30 & $-0,41$ & 1,67 & $3,50 \mathrm{~m}$ \\
Probe 23 & 0,67 & 0,53 & 0,28 & 1,01 & 2,60 & $3,20 \mathrm{~m}$ \\
Probe 24 & 0,58 & 0,55 & 0,30 & $-0,72$ & 1,97 & $2,80 \mathrm{~m}$ \\
Probe 25 & 0,89 & 0,43 & 0,19 & $-1,50$ & 4,54 & $2,35 \mathrm{~m}$ \\
Probe 26 & 1,01 & 0,24 & 0,06 & $-0,98$ & 9,94 & $1,90 \mathrm{~m}$ \\
Probe 27 & 1,24 & 0,20 & 0,04 & $-0,83$ & 9,10 & $1,45 \mathrm{~m}$ \\
Probe 28 & 1,05 & 0,21 & 0,04 & 0,25 & 6,11 & $1,00 \mathrm{~m}$ \\
Probe 29 & 0,85 & 0,28 & 0,08 & $-0,93$ & 5,45 & $0,55 \mathrm{~m}$
\end{tabular}

\subsubsection{Mosbacher Sande}

\begin{tabular}{lcccrr}
\hline Nr. & $\overline{\mathrm{X}}$ & $\sigma$ & $\sigma^{2}$ & $\alpha_{3}$ & \multicolumn{1}{c}{$\alpha_{4}$} \\
\hline Probe 1 & 1,11 & 0,16 & 0,02 & 0,62 & 7,75 \\
Probe 2 & 1,07 & 0,13 & 0,02 & $-0,32$ & 7,34 \\
Probe 3 & 1,05 & 0,19 & 0,04 & $-1,59$ & 1,13 \\
Probe 4 & 1,06 & 0,13 & 0,02 & 0,17 & 6,60 \\
Probe 5 & 0,95 & 0,14 & 0,02 & 2,24 & 11,20 \\
Probe 6 & 1,09 & 0,15 & 0,02 & 0,25 & 9,70
\end{tabular}

